\title{
Evaluation of thermal diffusivity variations in multi-layered structures
}

\author{
by M.Hryciuk, A. Nowakowski
}

Gdansk University of Technology, Department of Medical nad Biological Electronics, ul. G. Narutowicza 11/12, 80-952 Gdansk, E-mail: hryci@biomed.eti.pg.gda.pl, antowak@eti.pg.gda.pl

\begin{abstract}
The paper deals with the problem how to reconstruct internal structure of tested multilayered biological objects using external heat flux. The procedures of nonlinnear least squares approximation are used for this purpose. Two methods of investigation are presented: 1. active dynamic thermography using a step heat excitation 2. an isothermal contact probe for heat flux measurement. Some results of examination of phantoms and a burned skin are described.
\end{abstract}

\section{Introduction}

Visualisation of delaminations, corrosion areas, moisture in buildings, cracks in fresco paintings and other superficial inhomogenities by use of infrared active dynamic thermography has already been widely described, e.g. QIRT. Quantification of measurement results is the problem which has been intensively investigated in recent years. Although sometimes the ability of visualisation of raw thermal responses or a simple arithmetical operation on two thermograms, like subtraction or contrast evaluation, gives satisfactory data, the information of thermal properties distribution would give insight into internal structure of a tested object. If such operation is possible for every pixel, we could get a 3-dimensional representation of the investigated structure. This may be called "thermal tomography". Several approaches to thermal examination of multilayered structures are already known: $[1,2,3]$.

Aim of this paper is evaluation of thermal diffusivity distribution in multilayered biological structures and study on limitations of the methods. The presented reconstruction algorithm is dedicated for structures of thermal properties varying only in the direction perpendicular to the surface (multilayered structures - 1D model). Although we can analyse thermal response for every pixel of the recorded sequence, thus achieving a 3D-image of object's thermal properties in X-Y-Z space, we must remember that the variation of thermal properties of the object must be smoother in the lateral than in the perpendicular direction. The assumptions are fulfilled for human skin.

\section{Experiment}

Here we concentrate only on one type of excitation - the Heaviside step. Two methods are applied: a constant heat flux switched on in $t=0$ (the recorded value is temperature of the object's surface), and a surface temperature step achieved by isothermal probe application (the recorded value is the heat flux necessary to maintain the constant surface temperature).

The constant heat flux is generated by a matrix of halogen lamps with reflectors. The basic parameters of the source are: electrical power $4 \times 5 \times 50 \mathrm{~W}=1000 \mathrm{~W}$, set-up time: 60 $\mathrm{ms}$, light flux intensity at $0.5 \mathrm{~m}$ distance: $1100 \mathrm{~W} / \mathrm{m}^{2}$. For black bodies the whole light turns into heat flux passing the object's surface. For non-black bodies one has to consider a limited emmisivity coefficient (both for the excitation and the IR-recorder spectra) and specific optical penetration depth, reaching $1 \mathrm{~mm}$ for the caucasian skin [4] (this means that the excitation involves not only the external surface). One of the solutions to increase 
the absorbsion coefficient is to paint the object's surface using a black paint or carbon dust. The thermal response (surface temperature of a tested object) is recorded with Agema 900 Thermovision camera.

For the surface temperature step change excitation a special contact heater has been designed (Fig.1). It is a thin copper route etched on epoxy laminate. Basic parameters are: diameter - 22mm; thickness - 0.5mm; copper path thickness - $18 \mu \mathrm{m}$; copper path width $50 \mu \mathrm{m}$, resistance at the room temperature - $50 \Omega$. The heater's temperature is stabilised by a bridge circuit. The heater's resistance drives the feedback loop, which controls power delivery, in order to make the bridge balanced (what happens when the heater's resistance is equal to the reference). The voltage on the heater is recorded by a dedicated acquisition system starting at the moment of mechanical contact with the examined object. The electrical power $\left(P=U^{2} / R_{\text {set }}\right)$ delivered to the heater, divided by its area is equal to the heat flux transmitted to the object (heat losses on the rear side have been highly reduced by proper insulation). Typically, the temperature of the heater is set at $42^{\circ} \mathrm{C}$. The method gives spatially averaged thermal answer from the whole contact surface, and therefore assumes 1D heat flow, suitable for multilayered structures. If to neglect the heat losses at the edge, the whole heat flux is directed inside the object, perpendicularly to its surface.

\section{Direct problem}

According to the assumption of 1D heat flow the governing equations are shown for a one-dimensional case. When no internal heat sources exist in a tested structure, the heat transfer may by described by the Fourier-Kirchhoff equation:

$$
\rho(x) \cdot c(x) \frac{\partial T}{\partial t}=\frac{\partial k(x)}{\partial x} \cdot \frac{\partial T}{\partial x}+k(x) \frac{\partial^{2} T}{\partial x^{2}}
$$

where $\rho(x), c(x)$ and $k(x)$ are distributions of mass density, specific heat and thermal conductivity, respectively.

The initial state of the object is defined as:

$$
T(x, 0)=T_{0}(x), \text { for } t=0, x \in\langle 0, I\rangle
$$

where $I$ is the depth of the region of interest.

Two types of boundary conditions on the external surface are considered:

- Neuman (constant heat flux $\Phi_{\text {exc }}$ ) boundary condition:

$$
k(0) \frac{\partial T(0, t)}{\partial x}=\text { const }=-\Phi_{e x c}, \text { for } t>0, x=0
$$

- Dirichlet (temperature step change to $T_{\text {exc }}$ ) boundary condition

$$
T(0, t)=\text { const }=T_{\text {exc }} \text {, for } t>0, x=0
$$

In our implementation the duration time of excitation (and response recording) is in the range of $20 \mathrm{~s}$. By this time the heat penetrates into the excited object not deeper than a few milimeters (assuming thermal properties typical for biological tissues). We assume that the depth of further investigations is limited to $5 \mathrm{~mm}$. In fact, it is meanless, what kind of boundary condition is applied for the rear-side, because there is no heat transfer on this depth in the considered time range. We could use either isothermal:

$$
T(I, t)=T_{0}(I) \text { for } t>0, x=I
$$

or insulated boundary condition: 


$$
\frac{\partial T(I, t)}{\partial x}=0 \text { for } t>0, x=I
$$

For specified boundary conditions we find the solution (spatial and temporal distribution of temperature within the sample) of eq.(1) numerically, by use of finite differences. After discretisation of time and space (typically: $\Delta x=0.1 \mathrm{~mm}, \Delta t=0.01 \mathrm{~s}$ ) eq.(1) becomes:

$$
\rho_{m} c_{m} \frac{T_{m}^{p+1}-T_{m}^{p}}{\Delta t}=\frac{k_{m+1}-k_{m}}{\Delta x} \frac{T_{m+1}^{p}-T_{m}^{p}}{\Delta x}+k_{m} \frac{T_{m+1}^{p}+T_{m-1}^{p}-2 T_{m}^{p}}{\Delta x^{2}}
$$

where superscripts refer to time and subscripts to space domain. When we consider the fact that in soft biological tissues volumetric heat capacity varies in much lower range that the thermal conductivity, and the fact that the first component of the sum in the right-handside of eq.(7) is much lower than the second one (especially in structures with gradual variation of parameters), we may use the following approximation:

$$
\frac{k_{m+1}-k_{m}}{\rho_{m} c_{m}} \approx \frac{k_{m+1}}{\rho_{m+1} c_{m+1}}-\frac{k_{m}}{\rho_{m} c_{m}}
$$

which gives:

$$
T_{m}^{p+1}=T_{m}^{p}+\frac{\Delta t}{\Delta x^{2}}\left[\left(\alpha_{m+1}-\alpha_{m}\right)\left(T_{m+1}^{p}-T_{m}^{p}\right)+\alpha_{m}\left(T_{m+1}^{p}+T_{m-1}^{p}-2 T_{m}^{p}\right)\right]
$$

where $\alpha=k /(\rho c)$ is thermal diffusivity in subsequent nodes.

Eq. (9) is an explicit formulation of unsteady temperature distribution in 1Dheterogeneous medium; the temperature distribution in a specified moment is a function of only two factors: the temperature distribution in the previous moment and distribution of thermal diffusivity within the object.

Discrete form of the surface boundary conditions become for the isothermal probe:

$$
T_{1}^{p}=\text { const }=T_{\text {exc }} \text { for } p>0
$$

and for constant heat flux:

$$
T_{1}^{p+1}=T_{1}^{p}+2 \alpha_{1}\left(\Phi_{\text {exc }} \frac{\Delta t}{k_{1} \Delta x}-\frac{\Delta t}{\Delta x^{2}}\left(T_{1}^{p}-T_{2}^{p}\right)\right) \text { for } p>0
$$

Eq. (11) comes from constitution that the surface flux heats up the outermost slice (which is only $\Delta x / 2$ thick, to increase the calculation accuracy near the surface [5]), and the rest of the energy is transferred to deeper slices:

$$
\Phi_{\text {exc }}=\rho_{1} c_{1} \frac{\Delta x}{2} \frac{T_{1}^{p+1}-T_{1}^{p}}{\Delta t}+k_{1} \frac{T_{1}^{p}-T_{2}^{p}}{\Delta x}
$$

The relations presented above have been implemented in the MATLAB environment. The script allows to define the structure of the object (number of slices, maximal depth of analysis, spatial distribution of thermal properties), initial temperature distribution, and excitation type and value. Spatial-temporal variation of the temperature is the solution, from which also internal fluxes may be easily calculated (equation analogical to eq.(12)). 


$$
\text { DIRECT PROBLEM : } \left.\begin{array}{c}
T_{0}(x) \\
\alpha(x) \\
\Phi_{\text {exc }} \text { or } T_{\text {exc }} \\
k(0)
\end{array}\right\} \Rightarrow T(x, t) \text { or } \Phi(0, t)
$$

The knowledge of thermal conductivity of the outermost slice $(k(0))$ is necessary:

- in the case of constant heat flux excitation - for conversion of the heat flux excitation to temperature (see eq.3)

- in the case of isothermal excitation - for recalculation of the heat flux in the surface slice from temperature profile.

The knowledge of $k(0)$ and $\alpha(x)$ implies knowledge of the thermal effusivity $\beta(0)=k(0) / \alpha(0)^{1 / 2}$, therefore there is no disagreement between statement (13) and the fact that in homogenous medium thermal response depends on thermal effusivity $\beta$.

An exemplary result of solving the direct problem is presented in Fig. 2.

\section{Inverse problem}

The reason why we implemented the algorithm described in the previous section in MATLAB is our intention to use it for inverse problems. From known excitation parameters and thermal response measurements we may reconstruct the distribution of thermal diffusivity within the object. Note that in the direct problem we calculate spatial-temporal distribution of temperature within the whole object; in the inverse problem we know only the temperature and heat flux within the external slice:

$$
\text { INVERSE PROBLEM: } \left.\begin{array}{c}
T_{0}(x) \\
\Phi_{\text {exc }} \text { or } T_{\text {exc }} \\
T(0, t) \text { or } \Phi(0, t) \\
k(0)
\end{array}\right\} \Rightarrow \alpha(x)
$$

Such limitation of the available data makes the problem ill-posed; many combinations of $\alpha(x)$ may give similar thermal responses on the surface. Therefore to constrain a bit the reconstructive algorithm, we assume that in narrow areas the thermal properties of investigated objects are constant; from now we treat the investigated objects as multilayered structures with fixed layers number and thickness (of course several subsequent layers may have the same properties). Typically, we divide the $5 \mathrm{~mm}$ thick objects into 10 layers $(10 \times 0.5 \mathrm{~mm})$, each containing 5 differential slices (nodes) $(5 \times 0.1 \mathrm{~mm})$; see fig. 3 .

So, the reconstruction problem may be stated as: find the values of $\alpha_{\text {layer } 1}, \alpha_{\text {layer2 } 2, \ldots,}$ $\alpha_{\text {layerN }}(\mathrm{N}=$ number of layers $)$, which minimise the error function:

$$
T_{\text {err }}=\sum_{p=1}^{P}\left(T_{\text {meas }}^{p}-T_{\text {dir }}^{p}\left(\alpha_{\text {layer } 1}, \alpha_{\text {layer } \left.\left.1, \ldots, \alpha_{\text {layerN }}\right)\right)^{2}}\right.\right.
$$

for constant flux excitation, or:

$$
\Phi_{\text {err }}=\sum_{p=1}^{P}\left(\Phi_{\text {meas }}^{p}-\Phi_{\text {dir }}^{p}\left(\alpha_{\text {layer } 1}, \alpha_{\text {layer } 1, \ldots,}, \alpha_{\text {layerN }}\right)\right)^{2}
$$

for isothermal excitation. The superscript $p$ refers to discrete moments of time, meas refers to measured values (surface temperature or heat flux of the heater), dir - values calculated in direct problem algorithm for outermost slice, with pre-defined information about $T_{0}(x), k(0), \Phi_{\text {exc }}$ or $T_{\text {exc. }}$. This is done using the Isqnonlin function from MATLAB's optimisation toolbox. The function offers many options; in our case large scale algorithm is 
used. The a'priori knowledge of $T_{0}(x), k(0)($ actually $\beta(0))$ is successfully achieved by precalculation based on several first samples and approximation for homogenous material. For constant flux mode, affiliation of $T_{0}(x)$ and $k(0)$ into the optimised vector $\left.\left(\left[\alpha, k(0), T_{0}\right)\right]\right)$ gives better results.

In both methods, due to severe sensitivity to noise and artefacts, the following improvements were done:

- In the constant flux mode: The thermal camera is used in the line mode, which allows very fast acquisition (3472 lines/sec.). Every 35 lines are averaged, giving the efficient sampling rate of 100 lines/sec., what increases $S / N$ ratio by $15 \mathrm{~dB}$, and eliminates the effect of heat flux ripple coming from $50 \mathrm{~Hz}$ power supply. The disadvantage is the loss of one dimension (we can reconstruct only crossections of the object, not a 3D data). An alternative is space averaging, but this makes drastic reduction of resolution. Where possible, a reference object (made of the same material as the outermost layer of the investigated object) is put in the field of view. The difference between the theoretical curve (square root function fit in the measured data) and the measured one is used as a correction function added to every pixel's temperature.

- In the isothermal excitation mode: A polystyrene insulation of the rear side of the heater is used. An active ring decreasing the lateral heat outflow at the heater edges is under investigation. The data recorded during the first second is skipped for analysis (theoretically the heat flow reaches infinity at the moment of heater's application).

\section{Results and discussion}

Before measurements on living skin, preliminary reconstructions of phantoms have been done. One-, two-, and three-layered phantoms have been prepared using materials which have properties similar to biological tissues: rubber, silicone rubber, PVC, epoxide glue. Also a phantom with small inclusions has been constructed. Figures 4, 5 present exemplary results of phantom reconstruction. Measurements of healthy and burned skin of domestic pig have been done in-vivo. The background of the experiment is described in [8], and exemplary results are in Fig. 6.

With the improvements mentioned above both methods allow to reconstruct uniform multilayered structures up to $1 \mathrm{~mm}$ in depth. When additional constraints and longer time of acquisition are applied, even $2 \mathrm{~mm}$ deep objects may be analysed (fig. 4). In spite of the assumptions to restrict heat transfer to one-dimensional case, we achieved also a good structure reconstruction of phantoms with inclusions of small lateral size, because the temperature gradients are still much higher in perpendicular direction than in lateral (fig.5).

Both modes of examination seem to be useful for an objective method for skin burn diagnostics. The thermal approach has been already successfully used for this purpose [6] - using effective thermal effusivity, or calculating of a simple 4-parametric exponential model [7]. Medical aspects of changes presented in fig.6 will be discussed in [8].

There are two important problems to be solved for further improvement of the methods. First, results of the reconstruction procedure are highly dependent on the initial value of $\alpha$. The best results are achieved when the initial vector of $\alpha$ represents an uniform structure of properties equal to the outermost layer. When the initial $\alpha$ value is higher or lower by several percent, the reconstruction algorithm tends to give results of shifted absolute level, but of proper relationship of thermal diffusivity of layers. Larger changes of the initial value lead to reconstruction of improper structure, especially in deeper layers. Long time of calculation is the second problem. Even using of modern PC and compiled version of the script, reconstruction time takes several tens of minutes for every pixel. Due to the ill-posed nature of the problem, the time can not be reduced by enlargement of optimisation error or by enlargement of $\Delta x$. 


\section{REFERENCES}

[1] BALAGEAS D., KRAPEZ J.C., CIELO P., "Pulsed photo-thermal modelling of layered materials", J. Appl. Physics, vol 59, No2., 1986, p.348-357

[2] MACLACHAN J.W., AAMODT L.C., MURPHY J. C., "Time-resolved infrared radiometric imaging of coatings", Rev. Progr. Quant. Nondestr. Eval., vol . 8A, New York, 1989, p. 1297-1304

[3] VAVILOV A., MALDAGUE X., DUFORT B., "Adaptive thermal tomography algorithm", Proceedings of SPIE, vol 1933, Thermosense XV , Orlando 1993, p 166173

[4] ANDERSON R.R and PARRISH J.A, "The optics of the human skin" J. Invest. Dermatol., 77, 1981, p 13-19

[5] JANNA W.S., Engineering Heat Transfer, 2000, CRC

[6] BALAGEAS D.L., "Characterisation of living tissues from the measurement of thermal effusivity" Innov. Tech. Biol. Med., vol.12,1991

[7] NOWAKOWSKI A., et al. "Medical applications of model based dynamic thermography", Proceedings of SPIE, vol 4360, Thermosense XXIII , Orlando 2001, p 492-503

[8] HRYCIUK M, NOWAKOWSKI A., "Multi-layer thermal model of healthy and burned skin", Proceedings of EMBEC'02, Vienna, 2002 - in preparation

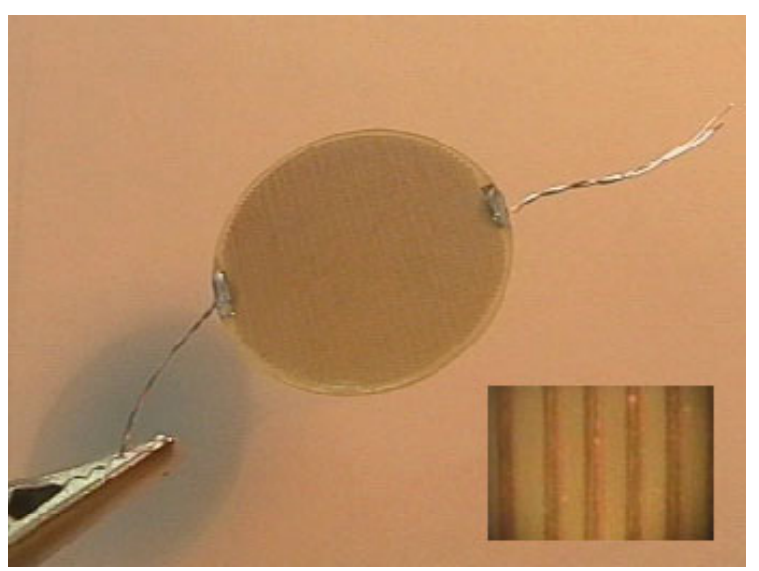

Fig. 1. Contact heater for temperature step excitation (in the corner: magnification of copper routes) 


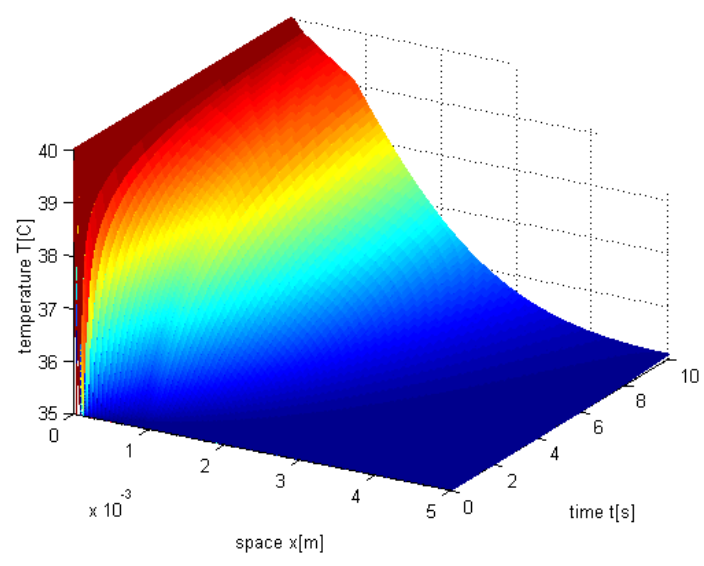

Fig. 2. Spatial-temporal distribution of thermal response of human skin for surface temperature step excitation (based on Stolvijk-Hardy model of the skin)
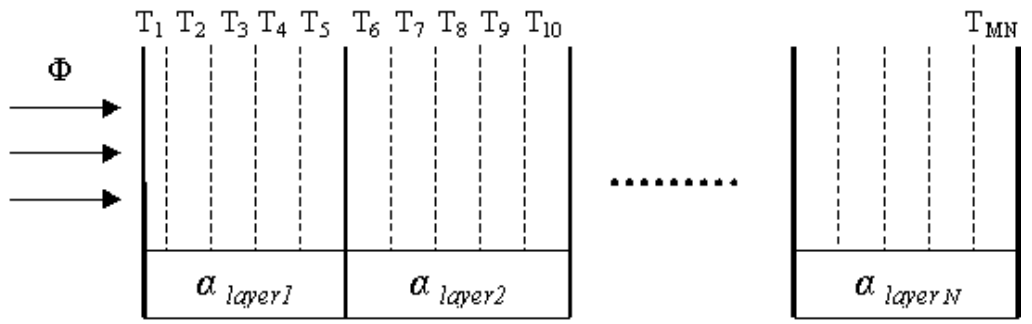

Fig. 3. Spatial discretisation of the model

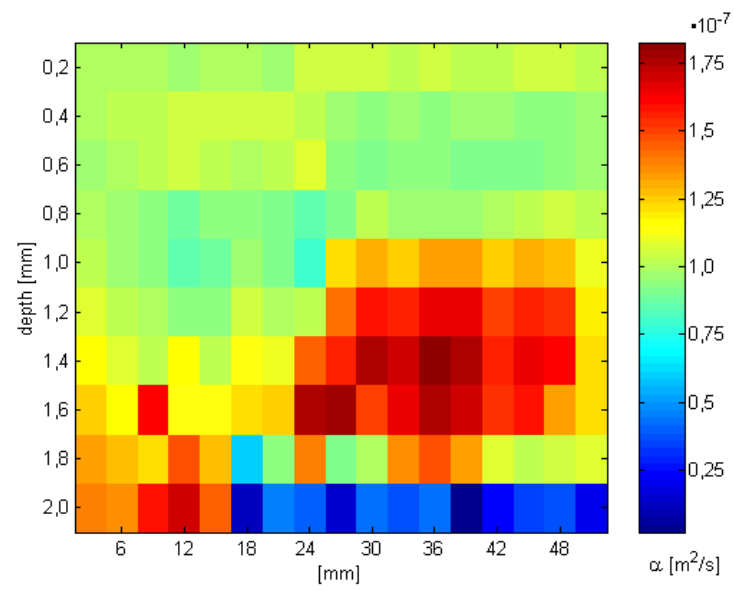

Fig. 4. Reconstructed structure of the phantom made of homogenous PVC $(0-27 \mathrm{~mm})$ and $1 \mathrm{~mm}$ deep PVC layer on epoxy glue substrate (27-54mm) 


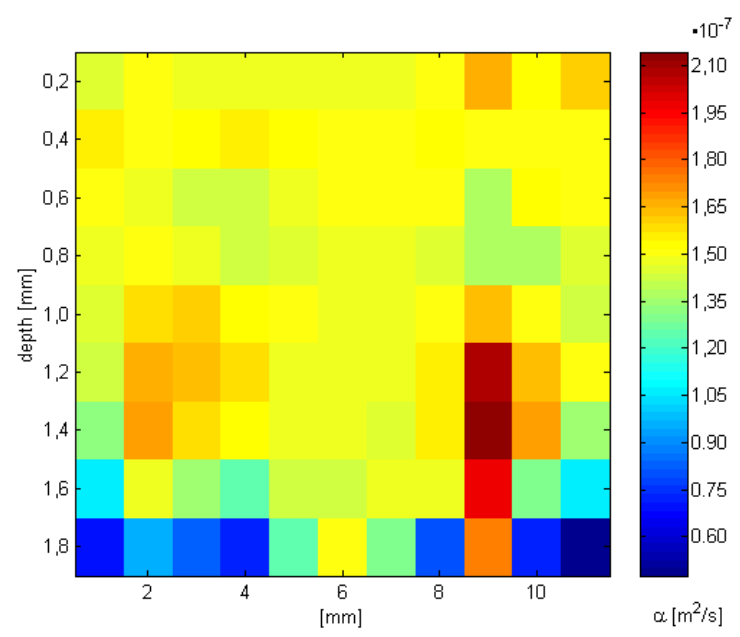

Fig. 5. Reconstructed structure of the phantom made of homogenous epoxy glue with two inclusions $\Phi 0,9 \mathrm{~mm} ; 1 \mathrm{~mm}$ deep (wood, graphite)

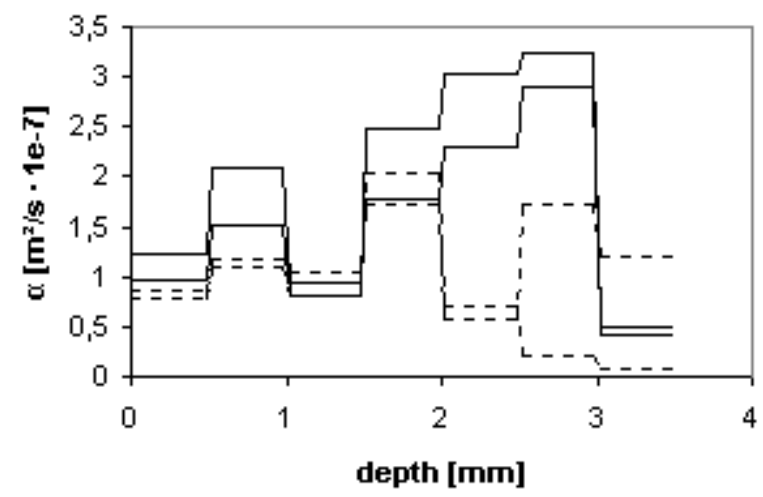

Fig. 6. Reconstructed structures of healthy (solid line) and 2-nd degree burned porcine skin (dotted line). Isothermal method. Data recorded on 3-rd day after injury 